\title{
Reducing the conservatism of the time domain passivity approach through consideration of energy reflection in delayed coupled network systems is
}

\author{
Michael Panzirsch $^{\mathrm{a}, *}$, Jee-Hwan Ryu ${ }^{\mathrm{b}}$, Manuel Ferre ${ }^{\mathrm{c}}$ \\ a Department for Analysis and Control of Advanced Robotic Systems, Institute of Robotics and Mechatronics, German Aerospace Center (DLR), Münchner Strasse 20, \\ Wessling 82234, Germany \\ ${ }^{\mathrm{b}}$ Biorobotics Laboratory, 307 Gajeon-ri Byeongcheon-myeon, Cheonan City, Chungnam Province, R. of Korea; School of Mechanical Engineering, Korea University of \\ Technology and Education, 330-708, R. of Korea \\ ${ }^{\mathrm{c}}$ Universidad Politécnica de Madrid, Centre for Automation and Robotics (CAR) UPM-CSIC, Escuela Tecnica Superior Ingenior, Jose Gutierrez Abascal 2, Madrid \\ 28006, Spain
}

\begin{abstract}
A B S T R A C T
The Time Domain Passivity Approach (TDPA), a method to inject adaptive damping to satisfy the passivity con- dition in real-time, has emerged as a

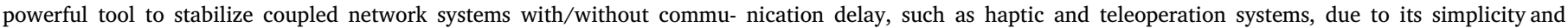

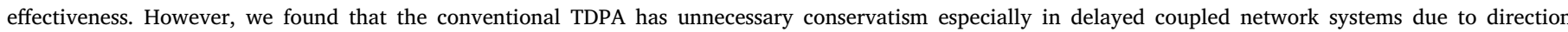

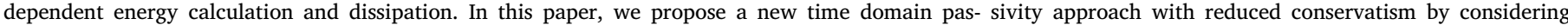

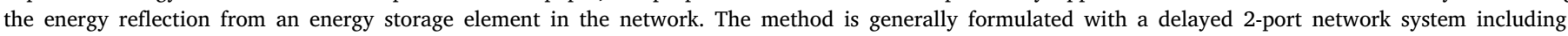
an energy storage element and implemented to a teleoperation system. The proposed method is experimentally tested and a comparison with the conventional TDPA reveals improved kinesthetic coupling and transparency in terms of position tracking and force reflection.
\end{abstract}

Keywords:

Time domain passivity control

Delayed coupled network system

Energy reflection

Teleoperation

\section{Introduction}

In recent years, there has been an increasing interest in time domain controllers that guarantee passivity online. In those methods, the sys tem's energy is monitored in real time and the controller gain is adap tively tuned in order to satisfy the passivity condition in the time domain. The Time Domain Passivity Approach (TDPA) has triggered this area of research. The authors of [1] proposed the TDPA for hap tic and coupled network systems. In [2], a time domain based method for passivity preserving discretization for haptic interaction was pro posed. Also focusing the interaction with virtual realities, the inertia matrix of a multi degree of freedom haptic device served the weight ing of a generalized variable passivity controller damping in [3]. In [4], the TDPA method was extended for teleoperation setups without delay. The TDPA for passivity control of the delay in a 2 Channel teleoperation system was introduced in [5]. The authors of [ 6 8 8] extended the TDPA for the application in more complex teleoperation architectures. In [9], the conventional TDPA has been applied to an industrial, multilateral, multi DoF teleoperation scenario.

Especially this type of online passivity guaranteeing controller has shown great advantages in stabilizing delayed coupled network systems.
Due to many unknown system parameters and the wide range of param eter variation in coupled network systems, their stabilization has been known to be quite complicated. A variety of control concepts as the wave variables method [10 12] were developed for delayed coupled network systems. The authors of [13] proposed a passive set point controller for time delayed teleoperation systems. An energy tank based two layer ap proach was introduced in [14]. Among them, TDPA has been getting more attention, and is recognized as one of the best performing control approaches compared to other types of controllers [15].

However, the TDPA has still been suffering from limited control per formance due to its controller design conservatism. To satisfy the passiv ity condition in real time, it often over sacrifices the performance of the controller which can result in large position synchronization error and feedback force attenuation [16]. Recently, researchers have proposed a modified method to reduce the design conservatism of the TDPA, in par ticular for improved position synchronization in delayed coupled net work systems such as teleoperation systems $[17,18]$.

However, we recently found that there is still large room to improve the performance of the TDPA especially for network systems with de layed position coupling. The conventional TDPA passivates the network considering a direction dependent energy flow observation and control due

\footnotetext{
4 This paper was recommended for publication by associate editor Dr. Mokrane Boudaoud.

* Corresponding author.

E-mail addresses: michael.panzirsch@dlr.de (M. Panzirsch), jhryu@kut.ac.kr (J.-H. Ryu), m.ferre@upm.es (M. Ferre).
} 
to the limitation of the considered passivity proof [16]. However, we found that this causes unnecessary conservatism in the controller de sign because it didn't allow us to differentiate the reflected energy from the pure transmitted energy in the 2 port network.

In this paper, we propose a new method reducing the design conser vatism of TDPA for coupled delayed network systems by avoiding the direction dependent energy monitoring. Therefore, a control loop in herent energy storage element is considered in the passivity controlled 2 port network. An ideal intended amount of energy in this energy stor age element is determined and the overall passivity is guaranteed by limiting the energy output of the 2 port to the ideal energy content of the energy storage element in a direction independent manner. As the result, the required amount of energy dissipation for preserving overall passivity is minimized by allowing energy reflection from the energy stor age element. It is important to note that, in contrast to [14] and [19], we consider potential energy but no dissipated energy in the energy stor age element to achieve a physically reasonable interpretation of energy reflection.

This paper is structured as follows: Section 2 presents the fundamen tals of Time Domain Passivity Control. Section 3 describes two state of the art approaches in TDPA of delayed 2 ports and the drawbacks resulting from the direction dependent energy monitoring. The pro posed control concept for delayed coupled network systems and the respective stability proof are presented in Section 4 . The advantages of the approach are discussed in a teleoperation setup employing ex periments in Section 5. A performance comparison with a state of the art approach is presented in Section 6. Finally, Section 7 provides the conclusion.

\section{Background}

This section reviews the two conventional Time Domain Passivity Approaches for delayed coupled network systems and shows the sources of possible conservatism of these approaches, resulting especially from the direction dependent energy observation.

To guarantee the stability of a system with states $\mathbf{x}$, the TDPA con siders the passivity criterion:

$V(\mathbf{x}(t))-V(\mathbf{x}(0)) \leq \int_{0}^{t} s(\mathbf{u}(\tau), \mathbf{y}(\tau)) d \tau=\int_{0}^{t} \mathbf{y}^{T}(\tau) \mathbf{u}(\tau) d \tau$.

As long as the energy increase $(V(\mathrm{x}(t))-V(\mathrm{x}(0)))$ in the system since $t=0$ is not higher than the integral of the power (supply rate $s$, input $\mathbf{u}$ and output y) that has entered the system, the system hasn't generated energy itself. In other words, the system is passive and thus absolute stability can be guaranteed [20].

The passivity criterion is highly modular in that the passivity of a sys tem is guaranteed if it consists of passive subsystems. The subsystems of a system can be designed with the help of the network representation. A network representation with clear causality and with a clear definition of the energy flow direction is essential for passivity based control meth ods as the TDPA. Especially in case of delayed coupled network systems, like time delayed teleoperation systems, the design of a proper network representation was not a trivial problem [6,8]. Figs. 1 and 2 show a 1 port and 2 port network, respectively. At each port, a power conju gate pair of effort variable (force) and flow variable (velocity) can be defined and measured. By integrating this power conjugate pair over time, the total energy can be calculated. Also, considering the sign of the conjugate pair, the energy flow direction can be determined.

A network representation of a delayed coupled network system is depicted in Fig. 3. The electrical models of human operator $h$, master device $m$, controller $c$, slave $s$ and environment $e$ consist of mass $M$ (in ductance), stiffness $K$ (capacitor) and damping $B$ (resistor) elements. The TDPA that can handle variable delay, jitter as well as package loss $[5,21]$, assures the passivity of the communication channel (CC) through

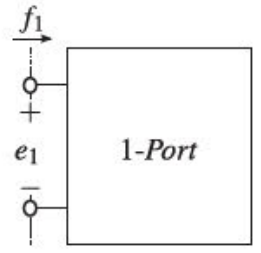

Fig. 1. 1-Port Network.

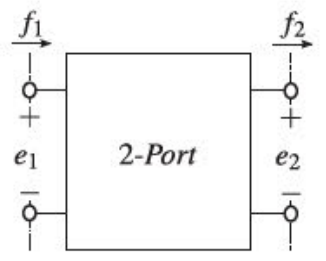

Fig. 2. 2-Port Network. so called passivity controllers (PC). The PCs terminate the passivity con trolled delayed 2 port of the CC (port 2 and port 3 ).

Note that in a delay free system, the passivity criterion is not a conservative method since generally an intrinsically passive position controller is applied. The terminations of the network, Agent $\Lambda 1$ and Agent $\Lambda 2$ have to be designed in a passive manner. In case of teleoper ation systems, Agent $\Lambda 1$ represents the human operator and the master device and Agent $\Lambda 2$ the slave in its environment. These terminations are obviously not passive but there is a widely accepted assumption that human operator and environment behave passive in their interaction such that the energy introduced by one is dissipated by the respective other. This assumption can be strengthened by the observation that, in real interactions, a human is used to use passive devices in active environments.

The direction of power flow (left to right L2R, right to left R2L) at a network subsystem port $i$ can be distinguished by the sign of the power in time step $\mathrm{k}$ :

$P_{i}^{L 2 R}(k)=\left\{\begin{array}{cc}0, & \text { if } P_{i}(k)<0 \\ P_{i}(k), & \text { if } P_{i}(k)>0\end{array}\right.$

$P_{i}^{R 2 L}(k)=\left\{\begin{array}{cc}0, & \text { if } P_{i}(k)>0 \\ -P_{i}(k), & \text { if } P_{i}(k)<0\end{array}\right.$

with the power $P_{i}$

$P_{i}(k)=v_{i}(k) F_{i}(k)$

calculated from the force $F_{i}$ and the velocity $v_{i}$ at port $i$. By integration over time, the respective energies $E_{i}^{L 2 R}(k)$ and $E_{i}^{R 2 L}(k)$ can be calcu lated:

$E_{i}^{L 2 R}(k)=T_{s} \sum_{j=0}^{k} P_{i}^{L 2 R}(j)$,

$E_{i}^{R 2 L}(k)=T_{s} \sum_{j=0}^{k} P_{i}^{R 2 L}(j)$,

with the sampling time $T_{s}$. The passivity criterion of a 2 port as depicted in Fig. 2 is

$E^{0}+E_{1}^{L 2 R}(k)+E_{2}^{R 2 L}(k) \geq E_{1}^{R 2 L}(k)+E_{2}^{L 2 R}(k)$

with the initial energy content $E^{0}$ of the 2 port network.

In the following, the TDPA control principles will be explained considering a basic teleoperation scheme. Fig. 4 presents a position computed force $\left(P F_{\text {comp }}\right)$ architecture in which a desired velocity (and position) and the computed controller force are exchanged through the communication channels which are affected by communication delay $T_{1}$ in $L 2 R$ and $T_{2}$ in $R 2 L$ direction. The PI coupling controller (Ctrl) which penalizes the velocity and position deviation of the two agents is located on the right side of the CC. In this case, the potential energy storage in the network results from the controller spring that aims the position synchronization of the coupled devices.

Fig. 5 presents the signal flow diagram of the TDPA [17] in a sim ple $P F_{\text {comp }}$ architecture. Through passivity observers (PO) on the left (L) and right (R) side of the CC, the energy behavior of the commu nication channel can be analyzed. The energy difference across a 2 port in one direction of energy flow can be observed and the energy 


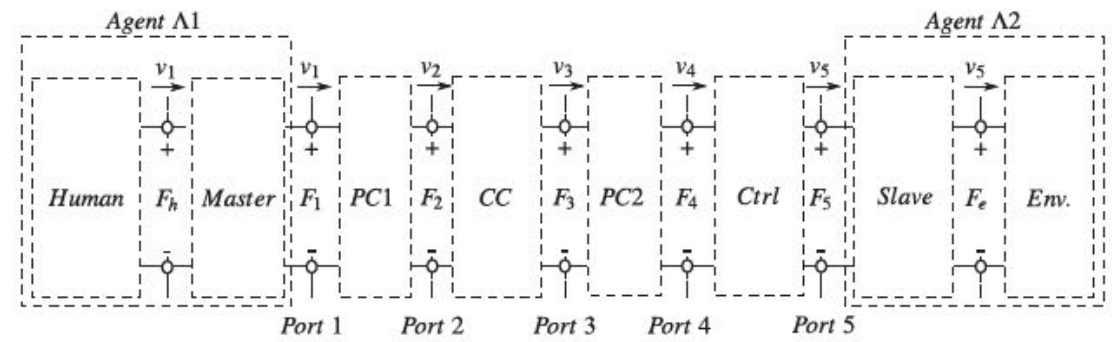

Fig. 3. Network Representation of a Delayed Coupled Network System.

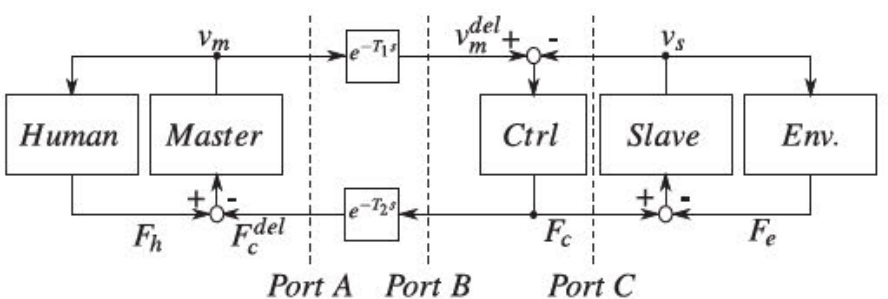

Fig. 4. Signal Flow Diagram of a $P F_{\text {comp }}$ Architecture.

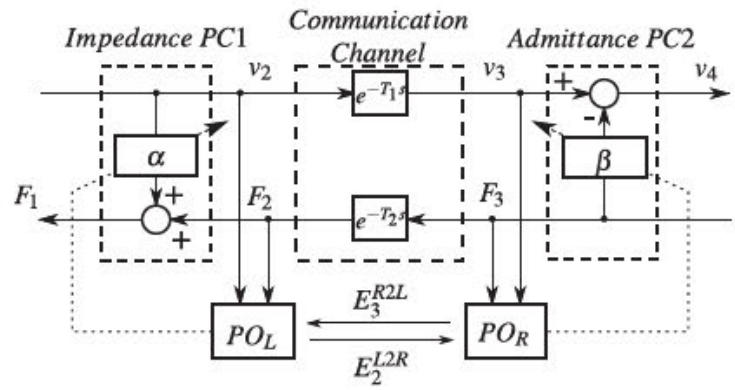

Fig. 5. Signal Flow Diagram of Time Domain Passivity Control for Approach 1.

$W^{P C 1}(k)$ and $W^{P C 2}(k)$ that has to be dissipated in time step $\mathrm{k}$ can be determined.

Passivity controller PC1 applies a variable damping to vary the force $F_{2}$ sent to the master device. At port 3, a velocity is sent in $L 2 R$ direction from Agent $\Lambda 1$ to the controller. Therefore, an admittance type PC2 is applied at port 3 in Approach 1 that injects a variable damping to vary this velocity signal. The depicted approach will be later referred to as Approach 1. Current implementations of time domain passivity control $[7,8,22]$ consider such a combination of impedance and admittance type controllers.

An admittance type PC dissipates the energy $W^{P C 2}(k)$ by a variation of the velocity $v_{3}$ :

$v_{4}(k)=v_{3}(k)-\beta(k) F_{3}(k) \quad$ with

$\beta(k)= \begin{cases}\frac{-W^{P C 2}(k)}{T_{S} F_{3}(k)^{2}} & \text { if } W^{P C 2}(k)<0 \\ 0 & \text { if } W^{P C 2}(k) \geq 0 .\end{cases}$ $F_{2}$ :

An impedance type PC dissipates energy by a variation of the force

$F_{1}(k)=F_{2}(k)+\alpha(k) v_{2}(k) \quad$ with

$\alpha(k)= \begin{cases}\frac{-W^{P C 1}(k)}{T_{S} v_{2}(k)^{2}} & \text { if } W^{P C 2}(k)<0 \\ 0 & \text { if } W^{P C 2}(k) \geq 0 .\end{cases}$

Without loss of generality, we can say that there is always at least one energy storage element in a coupled network system. The energy storage elements can be position coupling or force controllers and can be located on the left and/or the right side of the communication channel. Here, we focus on systems with a position coupling control element on the right side of the $\mathrm{CC}$ as it is applied in teleoperation systems with $P F_{\text {comp }}$ architecture. The adequacy of the proposed approach to other setups has to be further investigated in future. Still, the $P F_{\text {comp }}$ architecture can be applied in several delayed coupled networks with arbitrary agents that can be designed as passive subsystems. Note that in non teleoperation systems, a desired motion trajectory with open loop control may cause an energy input to the coupled network. But, this energy input can be considered in the supply rate $s$, that does not violate the passivity con dition (1). Therefore, the proposed approach can be applied to a large variety of applications.

\section{Problem definition}

Figs. 6 and 7 visualize how the conventional TDPA passivates de layed coupled network systems for two different ways of energy storage element consideration in the network. The Approach 1, presented in Fig. 6, excludes the energy storage element from the passivity analy sis based on the assumption that the energy storage element does not

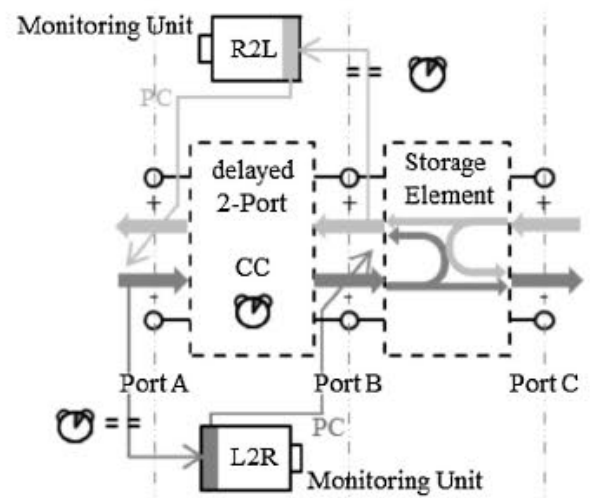

Fig. 6. Energy Observation and Passivity Control of Approach 1 [17].

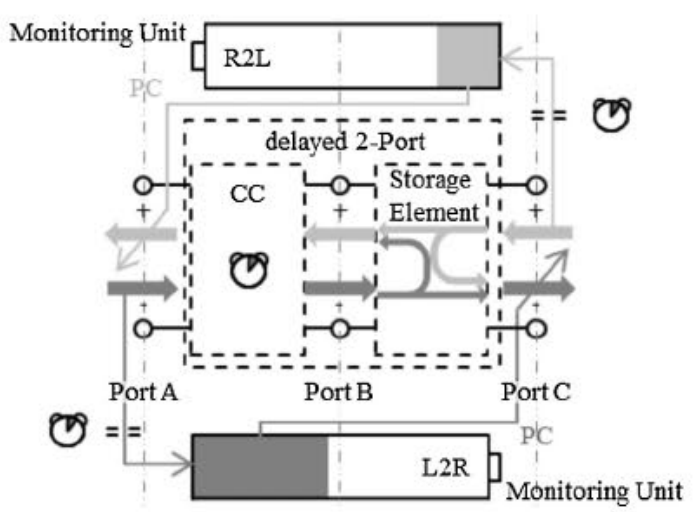

Fig. 7. Energy Observation and Passivity Control of Approach $2[5,24]$. 
break the passivity condition due to its intrinsic passivity. However, it prevents the consideration of the extra energy dissipation ability of the energy storage element and applies an admittance type PC which can lead to position drift. In contrast, the other conventional Approach 2 (see Fig. 7) includes the energy storage element in the passivity analysis and avoids admittance type PCs. For both cases, the TDPA is applied to dissipate an excessive amount of energy that is generated by the delayed 2 port, which potentially leads to instability.

\subsection{Functional principle of conventional approach 1}

In [17] (Approach 1, Fig. 6), two PCs terminate the delayed two port (port A, B) which contains the communication channel (CC). The energy flow in the system is presented by thick arrows that are marked with light gray (right to left: $R 2 L$ ) and dark gray color (left to right: $L 2 R$ ). The clock indicates delayed communication. In each direction of energy flow, an energy monitoring unit (battery like shape) is charged from the input power into the delayed 2 port (thin dark gray line at port $\mathrm{A}$ in $L 2 R$ direction and thin light gray line at port $\mathrm{B}$ in $R 2 L$ direction). The energy monitoring unit contains the available amount of energy that is allowed to leave at the respective output side of the delayed 2 port. On the output side, two passivity controllers (thin dark gray line at port $\mathrm{B}$ in $L 2 R$ direction and thin light gray line at port $\mathrm{A}$ in $R 2 L$ direction) assure via energy dissipation that not more energy than the available amount of energy in the respective monitoring unit leaves the 2 port in the respective direction. However, one interesting behavior of the energy storage element is that a part of the energy is transmitted through the storage element to the respective other side whereas the rest of the energy is reflected back as it is shown by the split arrows inside the storage element. Therefore, the $R 2 L$ energy flow at port $\mathrm{B}$ contains the reflected part of the energy from the $L 2 R$ energy flow at port $B$. The energy reflection and transmission will be analyzed in more detail in Section 3.3.

The network representation of a $P F_{\text {comp }}$ architecture with delay (com pare signal flow diagram of Fig. 4) for Approach 1 is presented in Fig. 3. Here, the position controller (Ctrl) represents the energy storage ele ment. Considering Fig. 3 and assuming a zero initial energy $E^{0}=0$, the passivity condition (7) is fulfilled, if

$E_{1}^{L 2 R}(k)+E_{4}^{R 2 L}(k) \geq E_{1}^{R 2 L}(k)+E_{4}^{L 2 R}(k)$.

Due to the delay, this condition has to be split into two parts

$E_{1}^{R 2 L}(k) \leq E_{4}^{R 2 L}\left(k-T_{2}\right)$

and

$E_{4}^{L 2 R}(k) \leq E_{1}^{L 2 R}\left(k-T_{1}\right)$.

Since the energies are monotonously increasing, conditions (13) and (14) fulfill condition (12). The passivity controller PC1 and PC2 of Ap proach 1 assure that condition (13) and (14) respectively are met. The energy that has to be dissipated can be calculated with

$W^{P C 1}(k)=E_{4}^{R 2 L}\left(k-T_{2}\right)-E_{1}^{R 2 L}(k)-W_{d i s s}^{P C 1}(k-1)$,

$W^{P C 2}(k)=E_{1}^{L 2 R}\left(k-T_{1}\right)-E_{4}^{L 2 R}(k)-W_{d i s s}^{P C 2}(k-1)$, where $W_{\text {diss }}^{P C i}(i \in\{1,2\})$ is the energy that has already been dissipated by the passivity controllers:

$W_{d i s s}^{P C i}(k-1)=W_{d i s s}^{P C i}(k-2)+W^{P C i}(k-1)$.

\subsection{Functional principle of conventional approach 2}

In contrast, in [5,23 25] (Approach 2, Fig. 7), the energy storage el ement is considered together with the CC in the passivity control. Thus, the two PCs are located on the left side of the CC (port A) and the right of the storage element (port $C$ ). Instead of the right side input into the CC (port B), the power input to the storage element on the right side (port C) is charged as the available amount of energy into the energy monitoring unit (battery like shape) in $R 2 L$ direction. Analogous to Ap proach 1, two PCs assure via energy dissipation that not more energy than available in the energy monitoring unit leaves the 2 port in the respective direction. Again, the energy dissipation of the PCs can be over conservative because the energy observation is direction depen dent and doesn't discriminate the directly reflected energy in the storage element.

Fig. 8 depicts the network representation of Approach 2. The PCs terminate the passivity controlled delayed 2 port including $\mathrm{CC}$ and the storage element (port 2 and port 4 ). These passivity controllers apply a variable damping to vary the forces sent in the respective directions (impedance type PC).

Analogous to Approach 1, the passivity controllers of Approach 2 assure that the conditions

$E_{1}^{R 2 L}(k) \leq E_{5}^{R 2 L}\left(k-T_{2}\right)$

and

$E_{5}^{L 2 R}(k) \leq E_{1}^{L 2 R}\left(k-T_{1}\right)$

are fulfilled which meets the passivity condition of the 2 port between port $\mathrm{A}$ and $\mathrm{C}$ with zero initial energy $E_{0}=0$ despite delay:

$E_{1}^{L 2 R}(k)+E_{5}^{R 2 L}(k) \geq E_{1}^{R 2 L}(k)+E_{5}^{L 2 R}(k)$.

The observed energies that need to be dissipated by the PCs are

$W^{P C 1}(k)=E_{5}^{R 2 L}\left(k-T_{2}\right)-E_{1}^{R 2 L}(k)-W_{\text {diss }}^{P C 1}(k-1)$,

$W^{P C 2}(k)=E_{1}^{L 2 R}\left(k-T_{1}\right)-E_{5}^{L 2 R}(k)-W_{\text {diss }}^{P C 2}(k-1)$.

Eq. (17) also holds for Approach 2.

\subsection{Analysis of drawbacks of the conventional approaches 1 and 2}

As mentioned before, both methods are conservative since the re flection of energy by the energy storage element is not considered. In the following, these limitations are further analyzed in a teleoperation example.

A free motion and wall contact in this system and the respective observed energies are presented in Fig. 9. The delay was set to zero in this experiment. The potential energy of the controller as the en ergy storage element can be charged up from both sides ( master $=$ Agent
Agent $\Lambda 1$

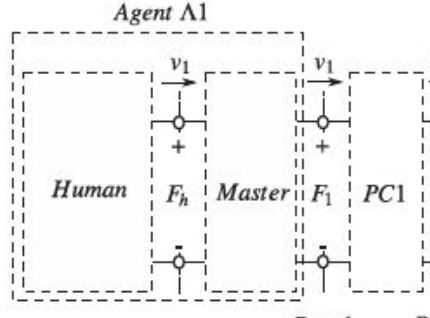

Port 1

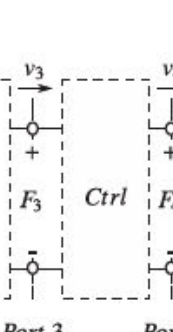

Port 3

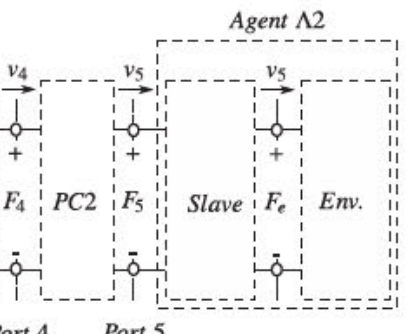

Port 5
Fig. 8. Network Representation of Approach 2 and 3. 

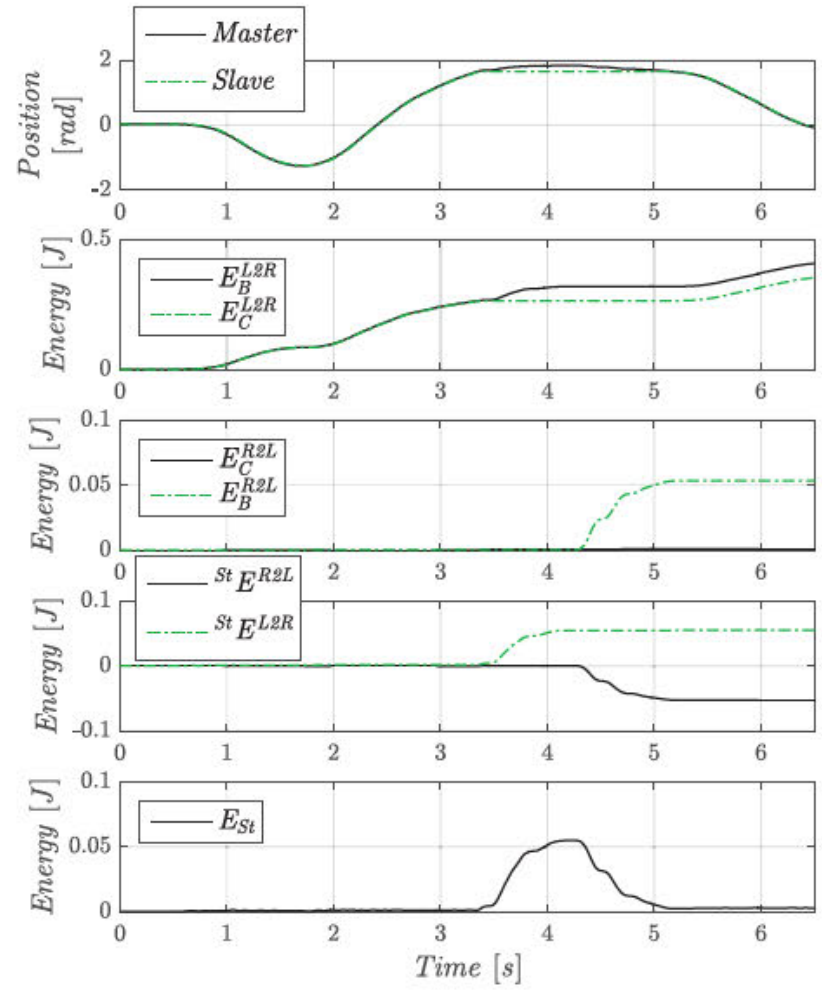

Fig. 9. Energy Behavior of a Controller with Storage Element.

$\Lambda 1$ and slave $=$ Agent $\Lambda 2$ ). In this bilateral experiment with $P F_{\text {comp }}$ ar chitecture, the master controls the slave in free motion until the slave touches a rigid wall $\left(K_{e} \approx \infty, B_{e} \approx 0\right)$ at about $t=3.4 s$. During free motion $\left(K_{e}=0, B_{e} \approx 0\right)$, the master injects the energy $E_{B}^{L 2 R}$ into the controller (storage element) at port B in $L 2 R$ direction which leaves this storage element mainly as $E_{C}^{L 2 R}$ such that the slave is moved. Therefore, the energy storage of the controller $E_{S t}$ is almost empty during free motion $(t=[0 s, 3.4 s])$.

When the slave touches the wall $(t=[3.4 s, 5 s])$, no energy can flow on the side of the slave since its velocity is zero (constant $E_{C}^{L 2 R}$ and $E_{C}^{R 2 L}$ ). With the wall penetration of the master, the energy storage $E_{S t}$ is filled up by energy $E_{B}^{L 2 R}$. When the master moves out of the contact, the energy of the storage is released as $E_{B}^{R 2 L}$ in $R 2 L$ direction to press the master out of the wall (energy reflection).

Mainly during wall contacts, or in general when the motion of one robot is hindered e.g. by obstacles, workspace limitations or high damp ing, energy might be reflected by the network element storing potential energy. Respecting the energetic behavior of the storage element, the state of the art approaches have the following conservatism:

- In Approach 1, the passivity controller at port B dissipates en ergy in $L 2 R$ direction (that would otherwise charge up the energy storage element) although this energy might be reflected back in $R 2 L$ direction (if the PC would be deactivated). This may lead to over conservative energy dissipation. Also, for example the force or position that is sent to the storage element from Agent $\Lambda 1$ is varied by the PC at port $B$. Thus, the charging of the energy storage element is lower than intended by Agent $\Lambda 1$ which can change the coupling behavior drastically, as among others posi tion drift can appear. The effect of the port B PC on the conser vatism of Approach 1 will be further analyzed in the experiments of Section 5 and the experimental comparison in Section 6.

- The energy plots ${ }^{S t} E^{R 2 L}$ and ${ }^{S t} E^{L 2 R}$ in Fig. 9 serve the analysis of the conservatism of Approach 2. The charging of the spring by $E_{B}^{L 2 R}$ is considered as an energy dissipation ${ }^{S t} E^{L 2 R}$ by the en ergy storage element in Approach 2 since no power leaves the energy storage element during the wall contact at port $C\left(E_{C}^{L 2 R}\right)$. In contrast, the release of energy ${ }^{S t} E^{R 2 L}$ to the master in $R 2 L$ direc tion is observed as an energy generation since no power enters at port $C\left(E_{C}^{R 2 L}\right)$ during the wall contact. This energy which is wrongly interpreted as generated energy ${ }^{S t} E^{R 2 L}$ is dissipated by the PCs since the dissipation ${ }^{S}{ } E^{L 2 R}$ happens in the other direction of energy flow and therefore does not compensate for the energy generation. Thus, the power flow direction dependent analysis of Approach 2 results in high dissipation and high conservatism. Also, the performance of Approach 2 is analyzed in detail in the experiments of Section 5 .

\section{Proposed method considering energy reflection}

Considering the drawbacks of the conventional TDPA methods as ex plained in Section 3, this section proposes a less conservative method by considering the energy reflection at the potential energy storage element.

Fig. 10 visualizes the basic idea of the proposed method applied to a delayed coupled network system. The main motivation of this pro posed method is that we can guarantee overall passivity without being restricted by the direction of energy flow. Therefore, we bound the out put energy of the 2 port by the ideal intended amount of input energy to the energy storage element from the coupled passive system around it (terminations of the 2 port). Similar to Approach 2 in Section 3, two PCs are located at port $\mathrm{A}$ and port $\mathrm{C}$ in order to consider the energy storage element together with the $\mathrm{CC}$ in the delayed 2 port. Thus, the network representation of Approach 3 equals the one of Approach 2 presented in Fig. 8. However, in contrast to Approach 1 and 2, one unique available amount of energy is collected in the energy monitoring unit (battery like shape) collecting the energy input from both sides of the delayed 2 port. Since this monitoring unit has a physical relation to the energy storage element, the monitoring unit is located on the right side of the communication channel. Thus, the asymmetric setup of the physical sys tem is represented in the proposed approach. Locating the monitoring unit on the left side of the CC does not guarantee a stable system be havior due to the loss of this physical relation and the evaluation of this relocation remains for future work. The energy monitoring unit is charged up by the dark gray (port A) and light gray arrow (port C) rep resenting the power input in $L 2 R$ and $R 2 L$ direction, respectively. The energy observation at port $\mathrm{C}$ in this asymmetric structure guarantees the consideration of energy generation by the CC. This is described in more detail in Section 4.2. The dashed arrows indicate where the pas sivity controllers dissipate the excessive amount of energy considering the available energy in the monitoring unit. As explained later in more detail, the distribution of the dissipation to PC1 and PC2 depends on

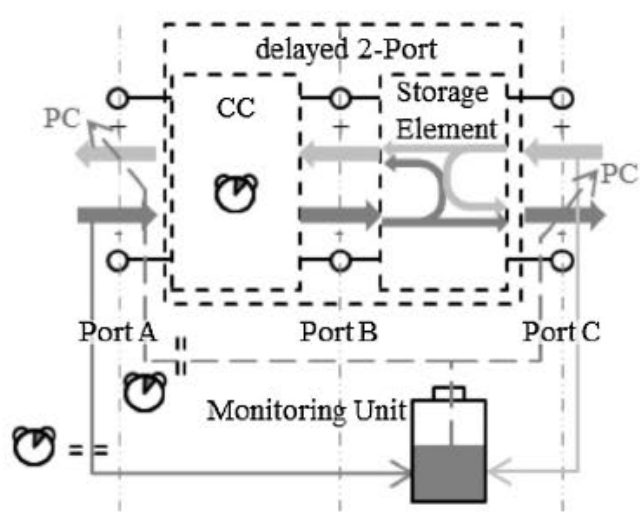

Fig. 10. Proposed Energy Observation and Passivity Control Considering Energy Reflection (Approach 3). 
the current distribution of output power of the energy storage element to port B and port C. Unlike the conventional TDPA, the unified energy monitoring unit allows a direction independent energy distribution re specting energy reflection.

In the following, the advantages of the proposed design and the con cept of the ideal energy storage in the monitoring unit are presented in Section 4.1 and Section 4.2 respectively. The detailed implementation is described in Section 4.3.

\subsection{Advantages over the conventional TDPA}

In contrast to Approach 1, the energy in Approach 3 can pass at port B to the energy storage element $(L 2 R)$ without being affected by a PC. Thus, the energy storage element that serves the desired coupling be havior is charged up as intended by the connected agents. This becomes clear when analyzing the analytical energy equation of the storage el ement: Exemplary, in the coupling through a position controller, the energy storage element equals a spring potential. This potential $E_{\text {pot }}$ can be calculated from the stiffness $K: E_{p o t}(t)=0.5 K\left(x_{L}^{*}(t)-x_{R}(t)\right)^{2}$, with in put $x_{L}^{*}$ from Agent $\Lambda 1$ and $x_{R}$ from Agent $\Lambda 2$. In Approach 1, the input $x_{L}^{*}$ is delayed and varied by the PC at port B whereas in the proposed Approach $3, x_{L}^{*}$ is only delayed. Therefore, in contrast to Approach 1, the energy storage element is charged up as intended by Agent $\Lambda 1$ in Approach 3.

Similar to Approach 2, the energy that is generated by the CC in $L 2 R$ direction and that crosses the storage element can be limited on the right side of the storage element (port $\mathrm{C}$ ). In contrast, energy that crosses the CC in $L 2 R$ direction and that is reflected by the storage ele ment back in $R 2 L$ direction is, in Approach 3, only affected by passivity control when it leaves the delayed 2 port on the left side. In Approach 1 , this reflected energy is passivity controlled on the way to the energy storage $(L 2 R)$ and on the way back $(R 2 L)$, which might lead to higher conservatism. The proposed concept has huge advantages concerning conservatism over Approach 2, where no reflection is considered since the energy behavior of the storage element is interpreted as a direction dependent combination of energy generation and dissipation.

\subsection{Ideal and real energy storage}

Note that the resulting energy content of the energy monitoring unit in Approach 3 (ideal storage) is not equal to the real energy content of the energy storage element, since that is affected by energy generation in the CC. Fig. 11 explains the difference between the ideal and real energy content in more detail. Since the active element CC adds energy in $L 2 R$ direction, the real storage (energy content of the energy storage element) which is filled up with the energies at Port 3 and Port 4 is equal to or higher than the ideal storage (energy content of the energy

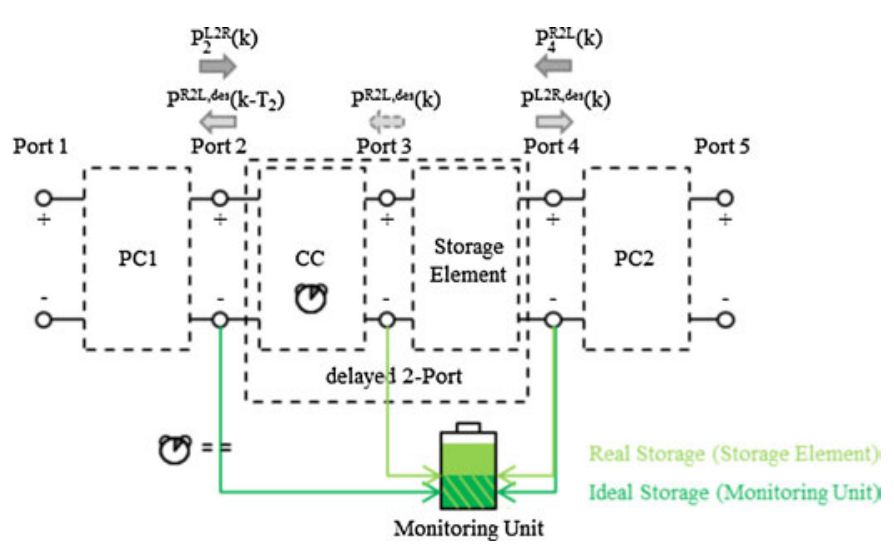

Fig. 11. Comparison of Real and Ideal Potential Energy Storages. monitoring unit). The energy monitoring unit collects the energy that is introduced from the agents into the 2 port between port 1 and 5 such that the ideal energy content can be regarded as the desired energy con tent of the controller (energy storage element). As depicted in Fig. 11, the ideal storage is built up by the power entering in $L 2 R$ direction at port $2\left(P_{2}^{L 2 R}\right)$ and in $R 2 L$ direction at port $4\left(P_{4}^{R 2 L}\right)$. As much energy as the ideal storage (energy monitoring unit) contains may leave at port 2 in $R 2 L$ and at port 4 in $L 2 R$ direction. Therefore, the allowed power out put $P^{L 2 R \text {, des }}$ and $P^{R 2 L \text {, des }}$ is calculated for the two directions with a logic that is described in Section 4.3. The limitation of the output power is assured by the passivity controllers $P C 1$ and $P C 2$. $P C 2$ limits the actual power output to $P^{L 2 R}$, des . The power $P^{R 2 L \text {, des }}$ is sent to the left side of

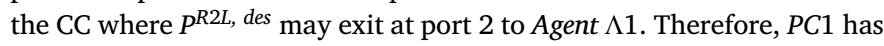
to consider the delayed desired power output $P^{R 2 L, d e s}\left(k-T_{2}\right)$ to limit $P_{2}^{R 2 L}(k)$.

\subsection{Implementation}

In contrast to the approaches with direction dependent dissipation Approach 1 and Approach 2, the passivity control is split up in two parts. In the first step, a limited, desired power output $P^{L 2 R, d e s}(k)$ and $P^{R 2 L, d e s}(k)$ of the energy storage element is determined and in the second step, the delay in $R 2 L$ direction is considered. Analogous to Approach 2 , the 2 port passivity condition of Approach 3 is

$E_{1}^{L 2 R}(k)+E_{5}^{R 2 L}(k) \geq E_{1}^{R 2 L}(k)+E_{5}^{L 2 R}(k)$.

The basic idea of the proposed concept is the consideration of the ideal energy storage $E_{S t}(k)$ in the energy monitoring unit which is built up by the energies $E_{2}^{L 2 R}(k)$ and $E_{4}^{R 2 L}(k)$. As described in Section 4.2, the avail able amount of energy that has to be accounted in the energy monitoring unit $E_{S t}$ can be determined by

$E_{S t}(k)=E_{S t}(k-1)+P_{2}^{L 2 R}\left(k-T_{1}\right) T_{s}+P_{4}^{R 2 L}(k) T_{s}$.

It is important to note that the power $P_{3}^{L 2 R}(k)$ measured at port 3 is not considered as an input, since it is affected by delay. But, since the energies are monotonously increasing and with the 2 port passivity con dition (7), it is clear that the delayed input energy from port 2 can be applied instead.

The passivity control is split into two parts: At first, the excessive amount of energy that leaves the energy storage element due to the energy generation of the CC in $L 2 R$ direction, has to be determined. Later, the allowed output power $P^{L 2 R \text {, des }}(k)$ and $P^{R 2 L \text {, des }}(k)$ that may leave the energy storage element has to be calculated.

1. The power that actually leaves the energy storage element at port 3 and 4 may lead to instability since additional energy may be injected into the storage element by the CC in $L 2 R$ direction. The actual output power $P_{\text {out }}^{\text {act }}(k)$ of the energy storage element in both directions of energy flow is:

$P_{\text {out }}^{\text {act }}(k)=P_{3}^{R 2 L}(k)+P_{4}^{L 2 R}(k)$.

If this power is smaller than or equal to the energy content of the energy monitoring unit $\left(P_{\text {out }}^{a c t}(k) T_{s} \leq E_{S t}(k)\right)$, this power may leave at the respective ports since it would not violate the pas sivity condition. Only if more power than available is exiting at port 3 or 4 , the power output of the energy storage element has to be limited. The excessive power output $P_{\text {exc }}(k)$ in both directions can be calculated as

$$
\begin{aligned}
& P_{\text {exc }}^{R 2 L}(k)=\left\{\begin{array}{cc}
P_{\text {exc }}(k) \frac{P_{3}^{R 2 L}(k)}{P_{\text {out }}^{\text {act }}(k)}, & \text { if } E_{S t}(k)<P_{\text {out }}^{\text {act }}(k) T_{s}, \\
0, & \text { if } E_{S t}(k)>P_{\text {out }}^{\text {act }}(k) T_{s}
\end{array}\right. \\
& P_{\text {exc }}^{L 2 R}(k)=\left\{\begin{array}{cc}
P_{\text {exc }}(k) \frac{P_{4}^{L 2 R}(k)}{P_{\text {out }}^{\text {act }}(k)}, & \text { if } E_{S t}(k)<P_{\text {out }}^{\text {act }}(k) T_{s}, \\
0, & \text { if } E_{S t}(k)>P_{\text {out }}^{\text {act }}(k) T_{s}
\end{array}\right.
\end{aligned}
$$


with the power $P_{\text {exc }}(k)$ that has to be dissipated in the current time step:

$$
P_{\text {exc }}(k)=E_{S t}(k) / T_{s}-P_{\text {out }}^{a c t}(k) \text {. }
$$

Here (Eqs. (26) and (27)), the dissipation of excessive energy is distributed to $L 2 R$ and $R 2 L$ direction proportionately to the real power output $P_{3}^{R 2 L}(k)$ and $P_{4}^{L 2 R}(k)$ in the respective direction. Although other distributions are conceivable, the chosen distri bution seems most reasonable since it is related to the current output power.

2. The excessive power $P_{\text {exc }}^{L 2 R}(k)$ can be directly subtracted from the power $P_{4}^{L 2 R}(k)$ by the right hand side $P C 2$ :

$$
\begin{aligned}
& P^{L 2 R, d e s}(k)=P_{4}^{L 2 R}(k)+P_{\text {exc }}^{L 2 R}(k), \\
& W^{P C 2}(k)=\sum_{j=0}^{k}\left(P^{L 2 R, d e s}(j)-P_{4}^{L 2 R}(j)\right) T_{s}-W_{d i s s}^{P C 2}(k-1) .
\end{aligned}
$$

The energy $W^{P C 2}(k)$ is then dissipated by $P C 2$ according to Eq. (11).

$P C 1$ has to assure that not more power than $P^{R 2 L, \text { des }}(k)$ with

$$
P^{R 2 L, d e s}(k)=P_{3}^{R 2 L}(k)+P_{\text {exc }}^{R 2 L}(k)
$$

leaves at port 2 in $R 2 L$ direction. Under consideration of the en ergy generation of the time delay in the communication channel, the observed energy of $P C 1$ can be calculated:

$$
W^{P C 1}(k)=\sum_{j=0}^{k-T_{2}} P^{R 2 L, d e s}(j) T_{s}-\sum_{j=0}^{k} P_{2}^{R 2 L}(j) T_{s}-W_{d i s s}^{P C 1}(k-1) .
$$

This energy $W^{P C 1}(k)$ is then dissipated by PC1 according to Eq. (11).

Impedance type PCs lead to forces with high frequencies due to sud den force changes. The authors of [16] proposed an additional passive virtual mass spring system to circumvent this effect. The virtual mass spring system acts as a low pass filter of force and velocity in both di rections in a way that passivity is maintained.

For the next time step, the available amount of energy in the en ergy monitoring unit $E_{S t}(k)$ has to be updated, as the powers $P_{3}^{R 2 L}(k)$ and $P_{4}^{L 2 R}(k)$ have exited at the respective ports of the energy storage element:

$$
\begin{aligned}
E_{S t}(k)= & E_{S t}(k-1)+\left(P_{2}^{L 2 R}\left(k-T_{1}\right)+P_{4}^{R 2 L}(k)\right. \\
& \left.-P_{3}^{R 2 L}(k)-P_{4}^{L 2 R}(k)\right) T_{s} .
\end{aligned}
$$

Note that $P_{3}^{R 2 L}(k)$ needs to be considered instead of $P_{2}^{R 2 L}\left(k-T_{2}\right)$ due to the delay.

\subsection{Passivity proof}

Fig. 12 presents the 4 steps (A D) of the passivity control approach that fulfill the passivity condition of the 2 port between port 1 and port 5. In step A of Eq. (24), the ideal reference storage is calculated that considers the input energies of the 2 port. The desired output powers are limited by the ideal storage $E_{S t}$ or the energy inputs $P_{2}^{L 2 R}$ and $P_{4}^{R 2 L}$ respectively

$$
\sum_{j=0}^{k} P^{R 2 L, d e s}(j) T_{s}+\sum_{j=0}^{k} P^{L 2 R, d e s}(j) T_{s} \leq \sum_{j=0}^{k-T_{1}} P_{2}^{L 2 R}(j) T_{s}+\sum_{j=0}^{k} P_{4}^{R 2 L}(j) T_{s}
$$

(compare Eqs. (26) (31)) in step B. Finally, in step C and D, the passivity controllers PC1 and PC2 assure that

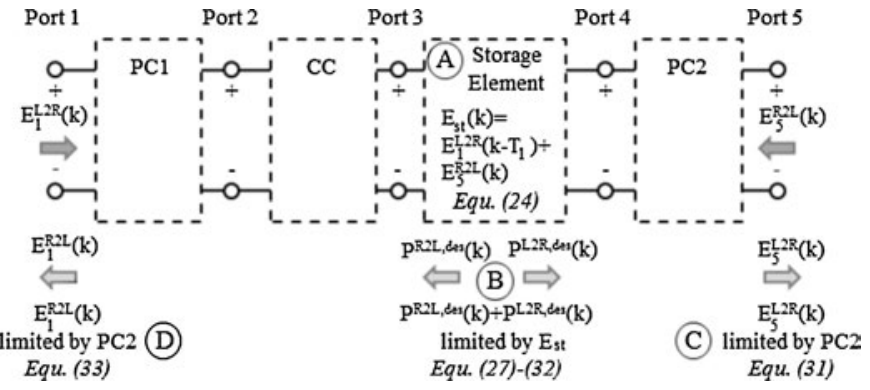

Fig. 12. Steps A to D of the Passivity Control.

$$
\begin{aligned}
& \text { - } E_{1}^{R 2 L}(k) \leq \sum_{j=0}^{k} P^{R 2 L, d e s}(j) T_{s} \quad \text { and } \\
& \text { - } E_{5}^{L 2 R}(k) \leq \sum_{j=0}^{k} P^{L 2 R, d e s}(j) T_{s} .
\end{aligned}
$$

Overall, assuming zero initial energy, the 2 port passivity condition (7) is fulfilled, since the outgoing energies are lower than the ingoing energies:

$E_{1}^{R 2 L}+E_{5}^{L 2 R} \leq E^{R 2 L, d e s}(k)+E^{L 2 R, d e s}(k) \leq E_{1}^{L 2 R}+E_{5}^{R 2 L}$.

Note that these energies are monotonously increasing.

\section{Application to teleoperation}

This section describes the application of the proposed Approach 3 to a teleoperation setup.

The following experiments serve the performance comparison of the three presented approaches. The experiments have been performed with the 1 DoF Master Slave System developed by SENSODRIVE GmbH (see Fig. 13). The control software ran on a real time system (QNX) at $1 \mathrm{kHz}$ sampling rate and was developed in Matlab/Simulink.

The first experiment Exp1 presents a free motion scenario without contacts at $200 \mathrm{~ms}$ roundtrip delay with Approach 1 (see Fig. 14). The port numbers are denoted according to Fig. 5 . At $t=[5 \mathrm{~s}, 7 \mathrm{~s}]$, it is obvi ous that the position synchronization is not perfect since Agent $\Lambda 2$ does not reach the maximum deflection of Agent $\Lambda 1$. This behavior results, to some extent, from the admittance type PC that causes a position drift. Note that the authors of $[17,18,26]$ proposed different methods to com pensate for the effect of position drift. Here, the concept of [17] was implemented. Still, due to the high delay, the compensation is limited.

The second experiment Exp2 in Fig. 15 shows a wall contact at $30 \mathrm{~ms}$ roundtrip delay in a setup with Approach 2. It can be analyzed at $t=[7 \mathrm{~s}, 7.5 \mathrm{~s}]$ that the feedback torque to Agent $\Lambda 1$ is reduced gravely when Agent $\Lambda 1$ moves out of the wall. This results from the wrong inter pretation of the energy reflection as an energy generation in Approach 2 (discussed in Section 3). In contrast to Approach 1, no position drift appears, since the PCs don't vary the input to the coupling controller in Approach 2.

The remaining experiments focus on the proposed concept (Approach 3). The system was tuned at the verge of stability at

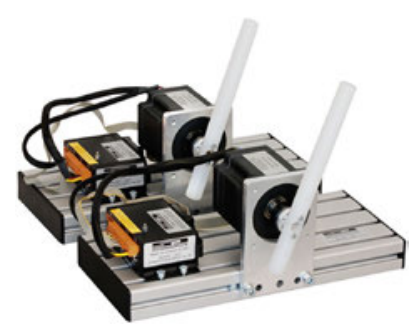

Fig. 13. 1-DoF Master-Slave-System. 

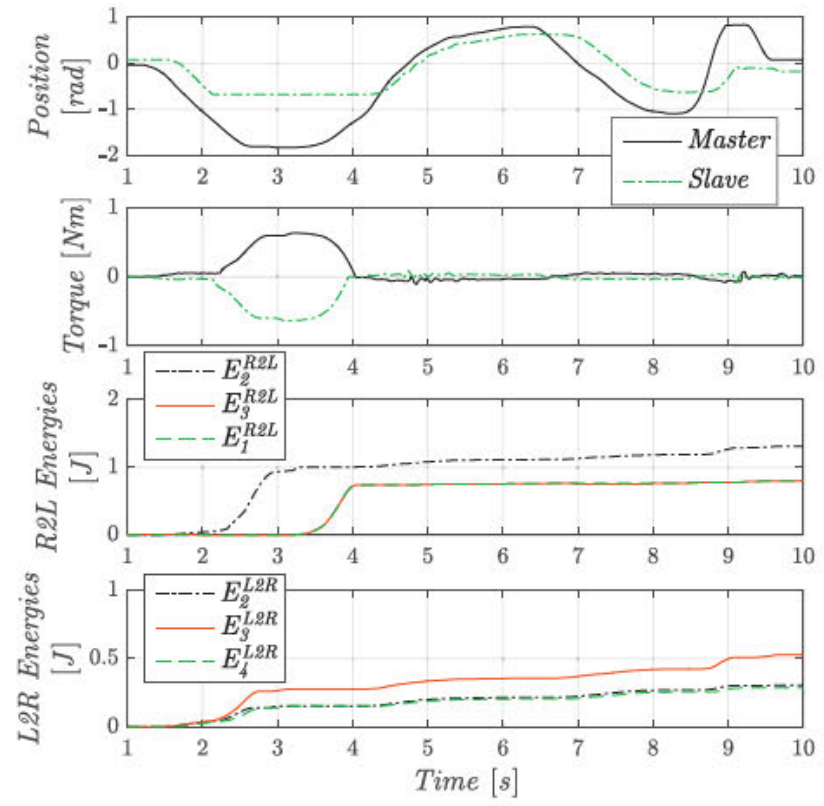

Fig. 14. Exp1: Free Motion and Wall Contact with $200 \mathrm{~ms}$ roundtrip delay in Approach 1.
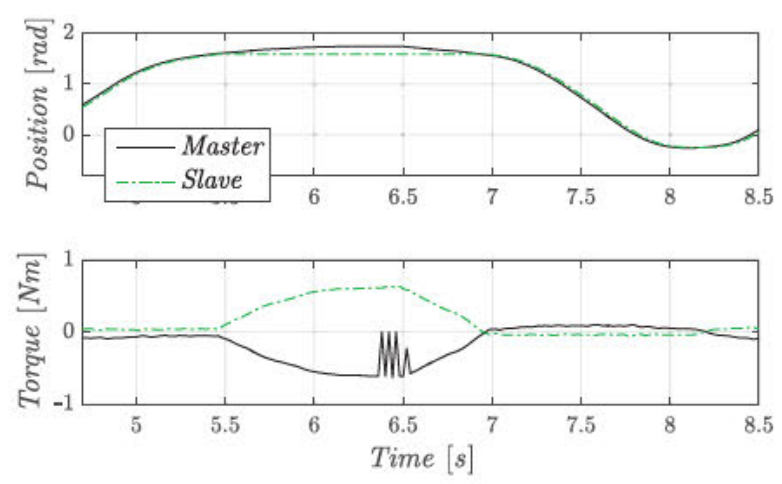

Fig. 15. Exp2: Wall Contact with 30ms Roundtrip-Delay in Approach 2.

$10 \mathrm{~ms}$ roundtrip delay (controller stiffness $K_{C}=2 \frac{\mathrm{Nm}}{\mathrm{rad}}$, controller damp ing $B_{C}=0.01 \frac{\mathrm{Nms}}{\mathrm{rad}}$ ). The third experiment Exp3 (see Fig. 16) presents the performance of the system by applying Approach 3 at $30 \mathrm{~ms}$ roundtrip delay. The position following of the two devices is satisfactory. The en ergy plot $E_{P P}$

$E_{P P}(k)=E_{1}^{L 2 R}(k)+E_{5}^{R 2 L}(k)-E_{1}^{R 2 L}(k)-E_{5}^{L 2 R}(k)$

(analogous to Eq. (7)) serves as the passivity proof of the PC controlled network between port 1 and port 5 (see Fig. 8). The charging and re leasing of the spring during the two wall contacts $(t=[9.3 \mathrm{~s}, 10.6 \mathrm{~s}]$ and $t=[12.7 \mathrm{~s}, 13.2 \mathrm{~s}])$ are clearly visible in the energy plot. Since $E_{P P}$ is never negative, the passivity during the operation is confirmed. There is only little PC dissipation during free motion. When the master moves the slave against the wall $(t=[9.3 \mathrm{~s}, 10.1 \mathrm{~s}])$, the storage is built up such that no power has to be dissipated since it is stored in the energy storage ele ment. When the master moves out of the wall $(t=[10.1 \mathrm{~s}, 10.7 \mathrm{~s}])$, power is flowing to the master such that mainly PC1 dissipates energy that was generated by the communication channel. Since the slave is steady dur ing a contact with a rigid wall, no power flows to the slave. Especially when the master moves out of the wall, the dissipation is much lower in Approach 3 compared to Approach 2 in Exp2. This can be analyzed
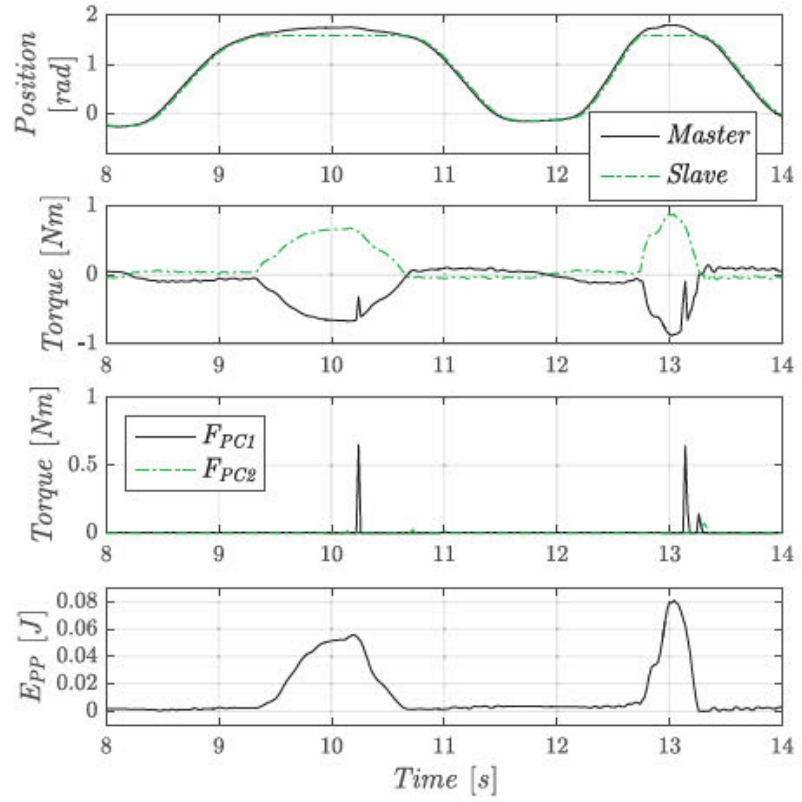

Fig. 16. Exp3: Wall Contact with $30 \mathrm{~ms}$ Roundtrip-Delay in Approach 3.
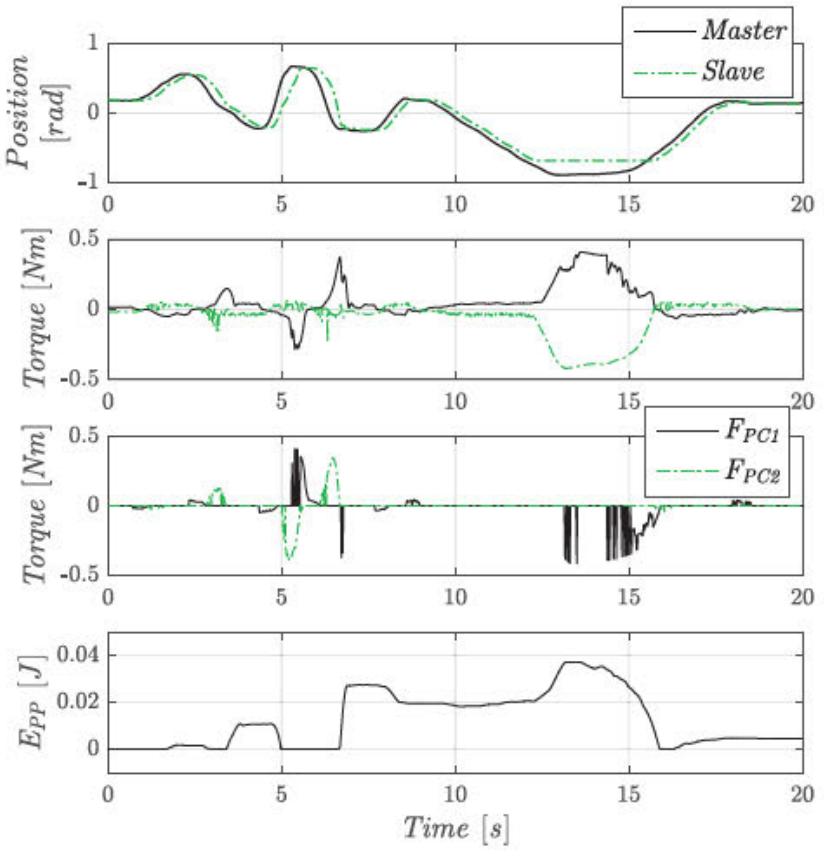

Fig. 17. Exp4: Free Motion and Wall Contact with $400 \mathrm{~ms}$ Roundtrip-Delay in Approach 3.

in the force feedback to the master which is more gravely reduced in Approach $2(t=[7.2 s, 7.5 s])$.

The fourth experiment Exp4 presents a free motion situation with slow $(t=[1 \mathrm{~s}, 3.5 \mathrm{~s}])$ and fast motion $(t=[3.5 \mathrm{~s}, 6.5 \mathrm{~s}])$ and a wall contact of the slave ( $t=[12.5 \mathrm{~s}, 15.5 \mathrm{~s}])$ at $400 \mathrm{~ms}$ roundtrip delay (see Fig. 17). The position following is clearly affected by the high delay. The energy plot $E_{P P}$ is always positive and thus confirms the passivity of the PC controlled network. Mainly passivity controller $P C 1$ is active during the wall contact. Very little energy needs to be dissipated in free motion at low velocities. In contrast to Approach 1 (Exp1), no position drift appears despite the high communication delay due to the consideration of energy reflection and the application of impedance type PCs. 


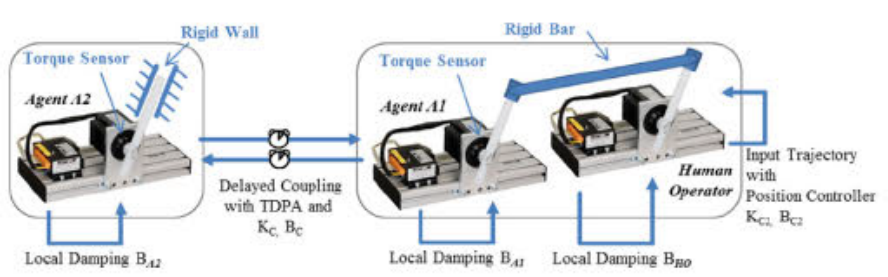

Fig. 18. Experimental Setup of Comparison Study.

\section{Experimental comparison study}

In this section, the time delay control method of [17] (Approach 1) and the proposed concept (Approach 3) are compared in a $P F_{\text {comp }}$ architecture with respect to different performance measures at vary ing delays. The analysis in Section 3.3 and especially the experiment in Fig. 15 show that Approach 2 has clear logical disadvantages over Approach 3 and is therefore excluded from the comparison.

Different metrics have been proposed to evaluate the performance of haptic devices and control approaches. For comparative studies, dif ferent performance indexes have been applied in $[27,28]$. The $\mathrm{Z}$ width and the M width were analyzed in [29] and [30] respectively. [31], [32] and [33] present performance evaluations of haptic interfaces and the respective control methods. The performance of common bilateral teleoperation concepts has been investigated in [34], [35], [36] and [37].

Here, similar to [38] that investigated the free space and stiffness transparency separately, free motion and wall contact scenarios are considered. Analogous to [15], the position error and the transmitted impedance are considered as performance measures.

\subsection{Experimental setup}

Two 1 DoF master slave systems have been applied as agent devices due to their low mass and low friction. To assure the reproducibility of the results and consistent system inputs, a human operator has been simulated with an additional $1 \mathrm{DoF}$ system connected with the Agent $\Lambda 1$ device through a rigid bar as presented in Fig. 18. In both approaches, a $P F_{\text {comp }}$ architecture was used. Since the research of $[17,18,26]$ showed that position drift compensation can improve the performance of Ap proach 1, the position drift compensation presented in [17] was applied to Approach 1. This setup allowed the measurement of interaction forces at Agent $\Lambda 1$ and $\Lambda 2$.

\subsection{Method}

A position controller $\left(K_{C 2}=2.3 \frac{\mathrm{Nm}}{\mathrm{rad}}, B_{\mathrm{C} 2}=0 \frac{\mathrm{Nms}}{\mathrm{rad}}, B_{\mathrm{HO}}=0.05 \frac{\mathrm{Nms}}{\mathrm{rad}}\right)$ acted on the device which replaced the human operator aiming at a si nusoidal motion with increasing frequency $f \in[0.2,1.4]$. A maximum frequency of $1.4 \mathrm{~Hz}$ has been chosen since a human operator should not move faster at high roundtrip delays to achieve a sufficient per formance. The approaches were analyzed for different roundtrip delays ( $T_{\mathrm{i}} \in\{30 \mathrm{~ms}, 100 \mathrm{~ms}, 200 \mathrm{~ms}, 400 \mathrm{~ms}\}$ ). The stiffness $K_{C}$ of the position controller connecting Agent $\Lambda 1$ and Agent $\Lambda 2$ was set to $K_{C}=2 \frac{\mathrm{Nm}}{\mathrm{rad}}$ in both approaches. This stiffness was chosen such that sufficient perfor mance was guaranteed up to $400 \mathrm{~ms}$ roundtrip delay. For the sake of comparability $K_{C}$ was constant throughout the comparative study. Indi vidual position controller damping $B_{C}$ and local damping gains $B_{\Lambda 1}$ and $B_{\Lambda 2}$ of the devices were adapted for each roundtrip delay and approach, to achieve (subjectively rated) the best performance regarding the spe cific roundtrip delay. Although the Time Domain Passivity controlled system is stable for any damping, the performance can be improved ap plying delay dependent damping values. Note that a local damping loop at the devices can be designed as an additional passive 2 port subsystem in the network such that it can be introduced into the presented control loops without violation of the passivity criterion. To consider different environmental impedances $Z_{e}$, the approaches were evaluated in a free motion $\left(Z_{e}=0\right)$ and a wall contact situation with fixed Agent $\Lambda 2$ device $\left(Z_{e} \approx \infty\right)$.

The metrics include the features denoted in Table 1 . In the wall con tact experiments, high effective stiffness values $M V\left(K_{\text {eff }}\right)$ are desired. In contrast, for free motion without environment contact, $M V\left(K_{\text {eff }}\right)$ should be zero. The position and path error should be low in both situations. The percentage of transmitted energy $\left(R A T_{E}\right)$ and the percentage of Agent $\Lambda 2$ forces $\left(M V\left(R A T_{F}\right)\right.$ ) should be close to one. In the wall contact scenario, the Agent $\Lambda 2$ does not move such that $M V\left(R A T_{F}\right)$ is considered instead of $R A T_{E}$. The path Path ${ }_{\Lambda 1}$ of Agent $\Lambda 1$ has to be analyzed in con text with the interaction force $F_{\Lambda 2}$ and the effective stiffness $K_{\text {eff. }}$ The dissipated energy $E_{\text {diss }}$ should be low.

\subsection{Results}

Figs. 19 to 22 depict the results for different environmental impedances and roundtrip delays at different frequencies. Since, in gen eral, the system performance at high delays is better at low frequency motions, the results of different frequency bandwidths are depicted.

In Fig. 19 (Exp5a), the free motion scenario at low input frequencies is depicted. At a roundtrip delay of $30 \mathrm{~ms}$, the dissipated energy and the
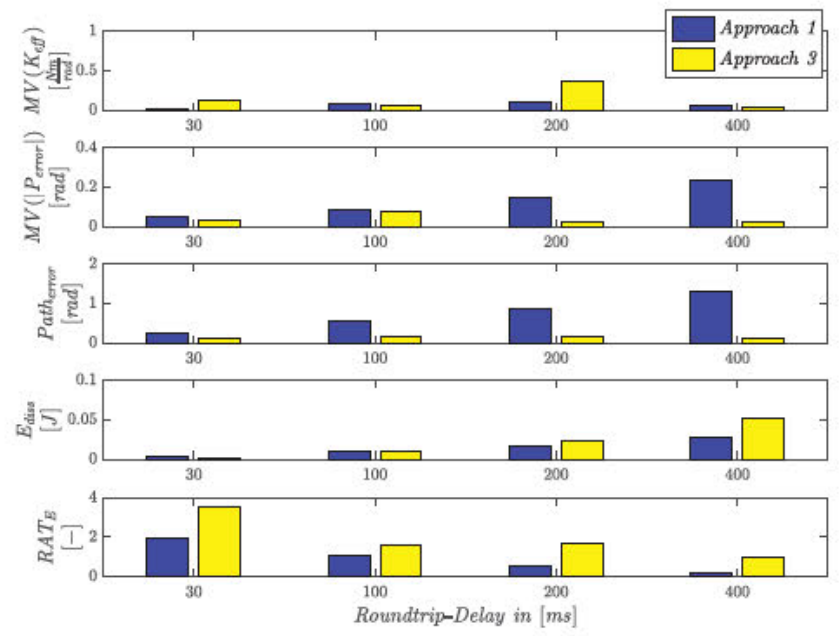

Fig. 19. Exp5a: Free Motion at $f=0.2 \mathrm{~Hz}$.

Table 1

Metrics.

\begin{tabular}{|c|c|}
\hline$M V\left(K_{e f f}\right)$ & mean effective impedance of evaluated frequencies $f \in[0.2 \mathrm{~Hz}, 1.4 \mathrm{~Hz}][15]$ \\
\hline Path arror & path error between the path length of the human operator motion and the motion of the Agent $\Lambda 2$ device \\
\hline Path $_{\mathrm{N} 1}$ & path $P_{a t h}$ of the Agent $\Lambda 1$ device \\
\hline$M V\left(\left|P_{\text {errar }}\right|\right)$ & mean position error between the Agent $\Lambda 2$ device and the delayed Agent $\Lambda 1$ device position \\
\hline$M V\left(\left|F_{\Lambda 2}\right|\right)$ & mean value of the absolute measured interaction forces of Agent $\Lambda 2$ \\
\hline$E_{\text {diss }}$ & sum of energy dissipated by the two passivity controllers $P C 1$ and $P C 2$ \\
\hline $\begin{array}{l}\text { RAT } \\
\text { MV }\left(R A T_{F}\right)\end{array}$ & $\begin{array}{l}\text { the ratio } R A T_{E}=\left(E_{\Lambda 1}+E_{\Lambda 2}\right) /\left(E_{\Lambda 1}^{K}+E_{\Lambda 2}^{K}\right) \text { of actual } E_{\Lambda i}(k)=\sum_{n=0}^{k}\left(v_{\Lambda i}(n) F_{\Lambda i}(n)\right)(i \in\{1,2\}) \text { and expected energy flow } E_{\Lambda i}^{K}=\sum_{n=0}^{k}\left(v_{\Lambda i}(n)\left(x_{\Lambda 2}(n)-x_{\Lambda 1}(n)\right) K_{C}\right) \\
\text { the mean value of the ratio } R A T_{F}=\left(F_{\Lambda 1}+F_{\Lambda 2}\right) / 2 F^{K} \text { of actual } F_{\Lambda i} \text { and expected computed force } F^{K}=\left(x_{\Lambda 2}(k)-x_{\Lambda 1}(k)\right) K_{C}\end{array}$ \\
\hline
\end{tabular}



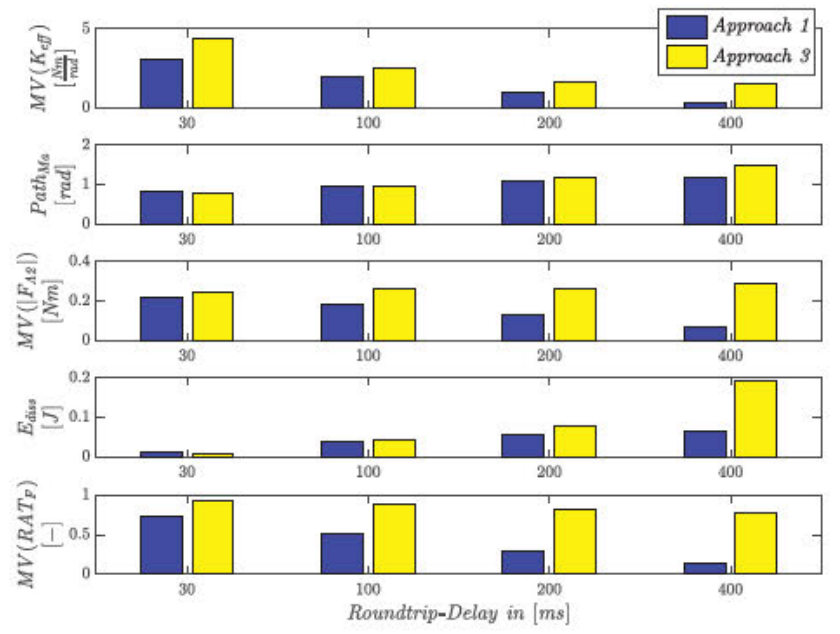

Fig. 20. Exp5b: Wall Contact at $f=0.2 \mathrm{~Hz}$.
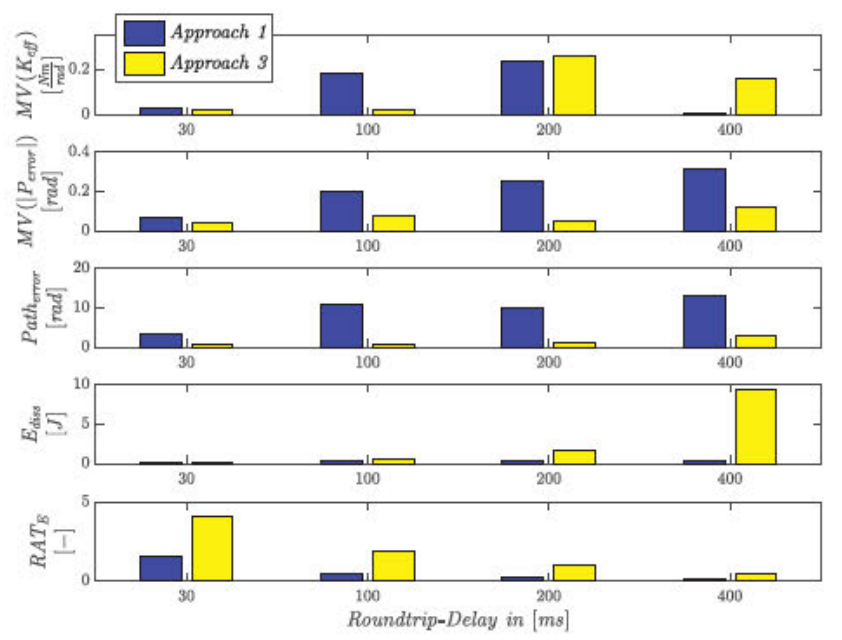

Fig. 21. Exp6a: Free Motion at $f \in[0.2 \mathrm{~Hz}-1.4 \mathrm{~Hz}]$.

position and path errors are lower for Approach 3. With increasing de lay, Approach 3 dissipates relatively more energy than Approach 1 but the position and path errors increase intensively in Approach 1 despite the slow motion. The big path error of Approach 1 results from position drift and reduces transparency strongly. Aside from the $200 \mathrm{~ms}$ condi tion, the effective stiffness is low in both approaches. The comparably high $M V\left(K_{\text {eff }}\right)$ of Approach 3 at $200 \mathrm{~ms}$ roundtrip delay may result from delay and frequency depending reflections in the communication chan nel. Note that this difference at $200 \mathrm{~ms}$ roundtrip delay vanishes when a wider range of frequencies is analyzed (Exp6a). $R A T_{E}$ has better values for Approach 3 at all delays.

In the wall contact situation at slow motion (see Fig. 20, Exp5b), the $M V\left(K_{\text {eff }}\right)$ is higher for Approach 3. Also, the metric $M V\left(\left|F_{\Lambda 2}\right|\right)$ indicates that the desired Agent $\Lambda 2$ motion results in higher Agent $\Lambda 2$ forces in Approach 3. The position drift of the admittance type PC in Approach 1 leads to a lower wall penetration of Agent $\Lambda 1$ (although the path of the Agent $\Lambda 1$ device is same for both approaches) and thus to lower interaction and coupling forces. Due to lower coupling forces, the dissi pated energy is reduced in Approach 1. $M V\left(R A T_{F}\right)$ has better values for Approach 3 at all delays.

The results at a bigger range of input frequencies in Fig. 21 (Exp6a) and Fig. 22 (Exp6b) are similar but less pronounced. Furthermore, the position and path errors increase for both approaches due to faster mo tions. Note that the path of Agent $\Lambda 1$ in the wall contact situation re
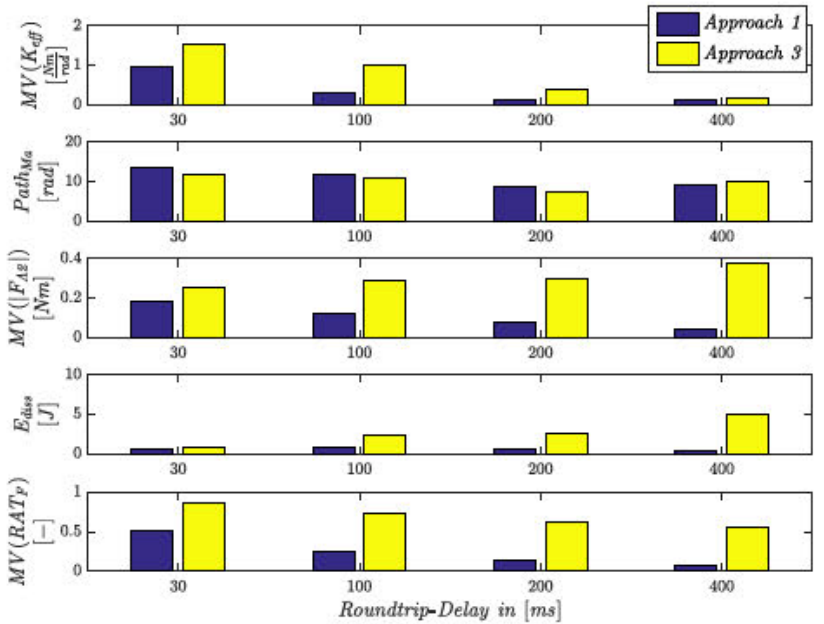

Fig. 22. Exp6b: Wall Contact at $f \in[0.2 \mathrm{~Hz}-1.4 \mathrm{~Hz}]$.

Table 2

Results.

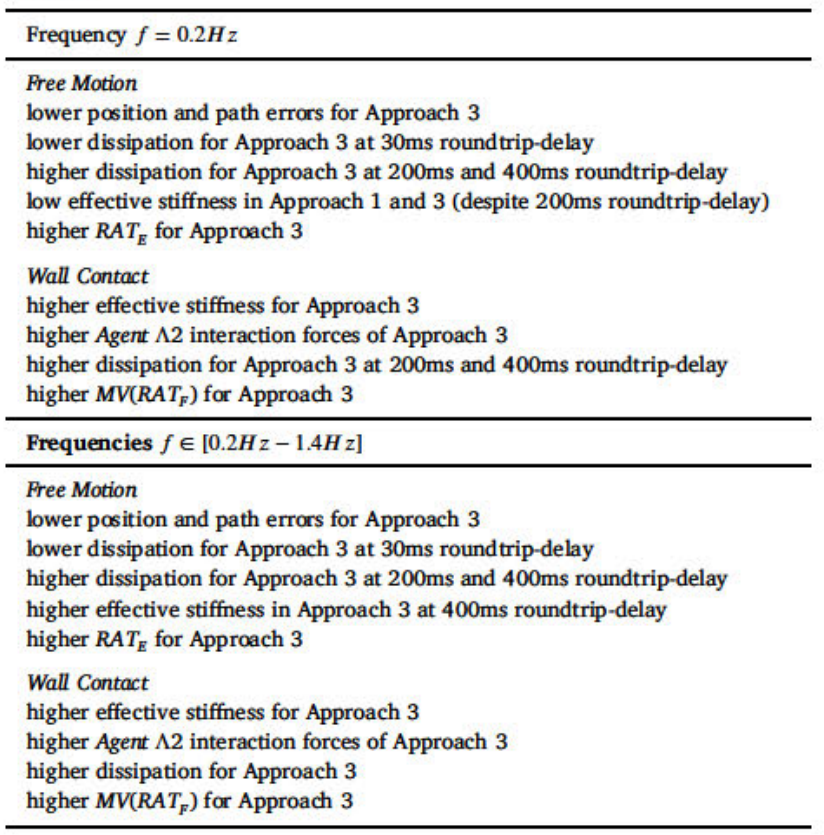

duces with the delay since the maximum frequency was set to $1.2 \mathrm{~Hz}$ for $100 \mathrm{~ms}$ roundtrip delay and to $1 \mathrm{~Hz}$ for $200 \mathrm{~ms}$ and $400 \mathrm{~ms}$ roundtrip delay. During free motion, the effective stiffness is lower for Approach 1 but this may result from position drift and can therefore not be consid ered as a benefit of Approach 1. Since the motion frequency is too high for the high roundtrip delays, during wall contact, the effective stiffness is lower than at $0.2 \mathrm{~Hz}$ motions in both approaches.

The main results are summarized in Table 2.

\subsection{Discussion}

In free motion, the position following is the most relevant criterion. The effective stiffness criterion provides lower accuracy in the free mo tion compared to the wall contact situation. Thus, considering free mo tion, Approach 3 with lower position and path errors promises better performance. At low roundtrip delay and slow motion, Approach 3 dis sipates less energy than Approach 1. The dissipated energy is not a suit able criterion since the energy amount of the system depends on the system behavior which differs strongly for the two approaches. Due to 
the position drift in Approach 1, the forces are lower. Lower forces in evitably lead to lower power flow in the system and thus to less dis sipation by the passivity controllers. Also, the relative dissipation of energy is not a reliable criterion. In contrast, the percentage of trans mitted energy which refers to the expected power flow resulting from the reference position and the controller constant $K_{C}$ is more reasonable. Also during wall contacts, Approach 3 provides better performance. This fact can be drawn from the higher effective stiffness and higher Agent $\Lambda 2$ interaction forces (compare $M V\left(R A T_{F}\right)$ and $M V\left(\left|F_{\Lambda 2}\right|\right)$ ) throughout all experiments with wall contact.

At motion inputs of higher frequencies $(f \in[0.2 \mathrm{~Hz}-1.4 \mathrm{~Hz}])$, the performance of both approaches decreases since the automated fre quency inputs do not react on the system behavior. In teleoperation setups, a human operator is able to adapt the velocity at high communi cation delays. To avoid large position drift in Approach 1 and to avoid high resistive forces due to position deviations in Approach 3, with in creasing delays, slower motions are required.

\section{Conclusion}

In this paper, a new time domain passivity control approach has been proposed that considers, in contrast to former approaches, energy reflec tion by an energy storage element. Thus, negative effects such as posi tion drift or excessive energy dissipation of former approaches could be avoided. Therefore, the proposed concept considers, in addition to the energy behavior of the communication channel, the potential energy of the coupling controller as an energy storage. Experiments showed good position synchronization of two coupled agents despite a round trip delay of $400 \mathrm{~ms}$. These results were strengthened in a comparison with a state of the art time domain passivity control approach consider ing different performance metrics. The proposed approach showed bet ter results considering position synchronization during free motion and transmitted impedances during wall contact at a wide range of commu nication delays. For future work, the proposed concept should also be integrated in a Position Position architecture or a 4 Channel framework and applied in more complex robotic applications.

\section{Acknowledgment}

The authors want to express their sincere gratitude towards Ribin Balachandran (DLR RM ARR) for his support in the evaluation of effec tive impedances.

\section{References}

[1] Hannaford B, Ryu J-H. Time-domain passivity control of haptic interfaces. Trans Robot Autom 2002;18(1):1-10.

[2] Stramigioli S, Secchi C, van der Schaft AJ, Fantuzzi C. A novel theory for sampled data system passivity. In: Proc. of 2002 IEEE/RSJ international conference on intelligent robots and systems, 2. IEEE; 2002. p. 1936-41.

[3] Hertkorn K, Hulin T, Kremer P, Preusche C, Hirzinger G. Time domain passivity control for multi-degree of freedom haptic devices with time delay. In: Proc. of 2010 IEEE international conference on robotics and automation. IEEE; 2010. p. 1313-19.

[4] Ryu J-H, Kwon D-S, Hannaford B. Stable teleoperation with time-domain passivity control. Trans Robot Autom 2004;20(2):365-73.

[5] Ryu J-H, Preusche C. Stable bilateral control of teleoperators under time-varying communication delay: time domain passivity approach. In: Proc. of 2007 IEEE international conference on international conference on robotics and automation. IEEE; 2007. p. 3508-13.

[6] Artigas J, Ryu J-H, Preusche C. Time domain passivity control for position-position teleoperation architectures. Presence 2010;19(5):482-97.

[7] Rebelo J, Schiele A. Time domain passivity controller for 4-channel time-delay bilateral teleoperation. Trans Haptics 2015;8(1):79-89.

[8] Artigas J, Balachandran R, Riecke C, Stelzer M, Weber B, Ryu J-H, et al. KONTUR-2: force-feedback teleoperation from the International space station. In: Proc. of 2016 IEEE international conference on international conference on robotics and automation; 2016. p. 1166-73.

[9] Panzirsch M, Balachandran RR, Weber B, Ferre M, Artigas J. Haptic augmentation for teleoperation through virtual grasping points. IEEE Trans Haptics 2018;11(3):400-16.
[10] Anderson RJ, Spong MW. Bilateral control of teleoperators with time delay. Trans Autom Control 1989;34(5):494-501.

[11] Niemeyer G, Slotine J-J. Stable adaptive teleoperation. J Ocean Eng 1991;16(1):152-62

[12] Yokokohji Y, Tsujioka T, Yoshikawa T. Bilateral control with time-varying delay including communication blackout. In: Proc. of 2002 symposium on haptic interfaces for virtual environment and teleoperator systems. IEEE; 2002. p. 285-92.

[13] Lee D, Spong MW. Passive bilateral teleoperation with constant time delay. Trans Robot 2006;22(2):269-81.

[14] Franken M, Stramigioli S, Misra S, Secchi C, Macchelli A. Bilateral telemanipulation with time delays: a two-layer approach combining passivity and transparency. Trans Robot 2011;27(4):741-56.

[15] Balachandran R, Artigas J, Mehmood U, Ryu J-H. Performance comparison of wave variable transformation and time domain passivity approaches for time-delayed teleoperation: preliminary results. In: Proc. of 2016 IEEE/RSJ international conference on intelligent robots and systems. IEEE; 2016. p. 410-17.

[16] Ryu J-H, Artigas J, Preusche C. A passive bilateral control scheme for a teleoperator with time-varying communication delay. Mechatronics 2010;20(7): 812-823.

[17] Artigas J, Ryu J-H, Preusche C. Position drift compensation in time domain passivity based teleoperation. In: Proc. of 2010 IEEE/RSJ international conference on intelligent robots and systems. IEEE; 2010. p. 4250-6.

[18] Chawda V, Van Quang H, O'Malley MK, Ryu J-H. Compensating position drift in time domain passivity approach based teleoperation. In: Proc. of 2014 haptics symposium. IEEE; 2014. p. 195-202.

[19] Lee D, Huang K. Passive position feedback over packet-switching communication network with varying-delay and packet-loss. In: Proc. of 2008 symposium on haptic interfaces for virtual environment and teleoperator systems. IEEE; 2008. p. 335-42.

[20] Fjallbrant T. Activity and stability of linear networks. IEEE Trans Circuit Theory 1965;12(1):12-17.

[21] Artigas J, Balachandran R, De Stefano M, Panzirsch M, Lampariello R, Albu-Schaeffer A, et al. Teleoperation for on-orbit servicing missions through the astra geostationary satellite. In: Proc. of 2016 IEEE aerospace conference. IEEE; 2016. p. 1-12.

[22] Panzirsch M, Balachandran R, Artigas J, Riecke C, Ferre M, Albu-Schaeffer A. Haptic intention augmentation for cooperative teleoperation. In: Proc. of 2016 IEEE International Conference on Robotics and Automation. IEEE; 2017. p. 5335-41.

[23] Panzirsch M, Artigas J, Tobergte A, Kotyczka P, Preusche C, Albu-Schaeffer A, et al. A peer-to-peer trilateral passivity control for delayed collaborative teleoperation. In: Haptics: perception, devices, mobility, and communication. Springer; 2012. p. 395-406.

[24] Panzirsch M, Artigas J, Ryu J-H, Ferre M. Multilateral control for delayed teleoperation. In: Proc. of 2013 international conference on advanced robotics; 2013. p. 1-6.

[25] Panzirsch M, Balachandran R, Artigas J. Cartesian task allocation for cooperative, multilateral teleoperation under time delay. In: Proc. of 2015 IEEE international conference on robotics and automation. IEEE; 2015. p. 312-17.

[26] Coelho A, Singh H, Muskardin T, Balachandran R, Kondak K. Smooth position-drift compensation for time domain passivity approach based teleoperation. In: Proc. of 2015 IEEE/RSJ international conference on intelligent robots and systems. IEEE; 2018 .

[27] Yokokohji Y, Yoshikawa T. Bilateral control of master-slave manipulators for ideal kinesthetic coupling-formulation and experiment. Trans Robot Autom 1994;10(5):605-20.

[28] Hayward V, Astley OR. Performance measures for haptic interfaces. In: Robotics research. Springer; 1996. p. 195-206.

[29] Colgate JE, Brown JM. Factors affecting the z-width of a haptic display. In: Proc of 1994 IEEE international conference on robotics and automation. IEEE; 1994 p. $3205-10$.

[30] Colonnese N, Okamura A. M-Width: stability and accuracy of haptic rendering of virtual mass. Robotics 2013;41.

[31] Hannaford B, Wood L, McAffee DA, Zak H. Performance evaluation of a six-axis generalized force-reflecting teleoperator. Trans Syst. Man Cybern. 1991;21(3):620-33.

[32] Adams RJ, Moreyra MR, Hannaford B. Stability and performance of haptic displays: Theory and experiments. In: International mechanical engineering congress and exhibition; 1998. p. 227-34.

[33] Radi M, Artigas J, Preusche C, Roth H. Transparency measurement of telepresence systems. In: Proc. of 2008 international conference on human haptic sensing and touch enabled computer applications. Springer; 2008. p. 766-75.

[34] Arcara P, Melchiorri C. Control schemes for teleoperation with time delay: a comparative study. Rob Auton Syst 2002;38(1):49-64.

[35] Aliaga I, Rubio A, Sanchez E. Experimental quantitative comparison of different control architectures for master-slave teleoperation. Trans Control Syst Technol 2004;12(1):2-11.

[36] Sankaranarayanan G, Hannaford B. Experimental comparison of internet haptic collaboration with time-delay compensation techniques. In: Proc. of 2008 IEEE international conference on robotics and automation. IEEE; 2008. p. 206-11.

[37] Rodríguez-Seda EJ, Lee D, Spong MW. Experimental comparison study of control architectures for bilateral teleoperators. Trans Robot 2009;25(6):1304-18.

[38] Willaert B, Reynaerts D, Van Brussel H, Vander Poorten EB. Bilateral teleoperation: quantifying the requirements for and restrictions of ideal transparency. Trans Control Syst Technol 2014;22(1):387-95. 


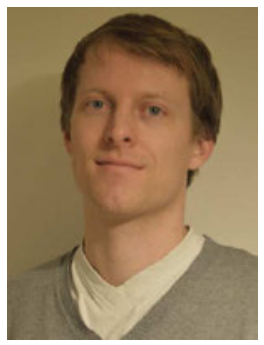

Michael Panzirsch was born in Donauwörth, Germany, in 1985. He received his Diploma in mechanical engineering from the Technical University of Munich (TUM) in 2010. Since then he is with the Department for Analysis and Control of Advanced Robotic Systems of the German Aerospace Center (DLR) in Oberpfaffenhofen as a researcher. In October 2018, he finished his $\mathrm{PhD}$ on multilateral teleoperation at the Universidad Politécnica de Madrid (UPM). His main areas of research interests are teleoperation of static and mobile robots, haptics and medical robotics.

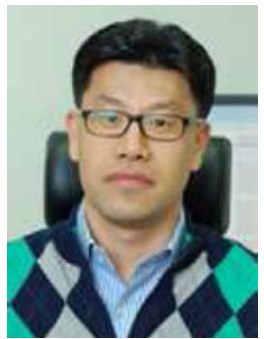

Jee-Hwan Ryu (M02) received the B.S. degree in mechanical engineering from Inha University, Incheon, South Korea, in 1995, and the M.S. and Ph.D. degrees in mechanical engineering from the Korea Advanced Institute of Science and Technology, Daejon, South Korea, in 1995 and 2002, respectively. He is currently a Professor with the Department of Mechanical Engineering, Korea University of Technology and Education, Cheonan, South Korea. His research interests include haptics, teleoperation, exoskeletons, and autonomous vehicles.

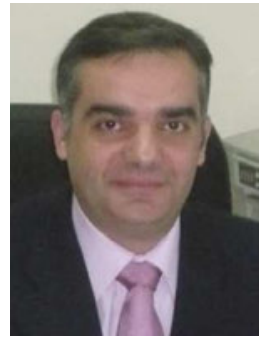

Manuel Ferre received the Laurea Degree in Control Engineering and Electronics in 1992 and PhD in Automation and Robotics in 1997, at the Universidad Politécnica de Madrid (UPM). In 1990, he started his research activity at this university, where he is currently a Profesor Titular since 2000. In 1997, he worked as a PostDoc in the Human-Machine System Laboratory of Massachusetts Institute of Technology (MIT). He has participated and coordinated several research projects in robotics and automatic control, both at national and international programs. His research interest is focused on automatic control, advanced telerobotics, and haptics. He has three patents of haptic devices and stereoscopic video cameras. He is author of more than 100 publications and editor of the 'Springer Series on Touch and Haptics System'. He is member of IEEE and serves as chair of the RAS Technical Committee on Telerobotics. He is also member of EuroHaptics Society where he serves as Treasurer. He has participated in International Program Committees of several conferences highlighting some editions of IROS and EuroHaptics. He was the chairman of the First Int. Workshop on Telerobotics in 2004 and hosted the EuroHaptics 2008 conference. 